\title{
APTIDÃO FÍSICA E LESÕES: 54 SEMANAS DE TREINAMENTO FÍSICO COM POLICIAIS MILITARES
}

\author{
PHYSICAL FITNESS AND INJURIES: 54 WEEKS OF PHYSICAL TRAINING AMONG MILITARY POLICE OFFICERS \\ APTITUD FÍSICA Y LESIONES: 54 SEMANAS DE ENTRENAMIENTO FISICO CON LA POLICÍA MILITAR
}

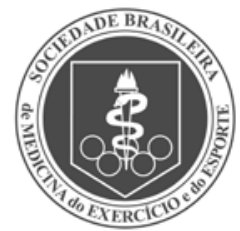

Artigo Original

Original ARtICLE

ARTíCULO ORIGINAL

\begin{abstract}
Lia Grego Muniz de Araújo' (Profissional de Educação Física)

Marcelo Sanches ${ }^{2}$ (Profissional de Educação Física)

Bruna Camilo Turi' (Profissional de Educação Física)

Henrique Luiz Monteiro 3

(Profissional de Educação Física)
\end{abstract}

\begin{abstract}
1. Universidade Estadual Paulista, Instituto de Biociências, Rio Claro, SP, Brasil.

2. Policia Militar do Estado de São Paulo, Bauru, SP, Brasil.

3. Universidade Estadual Paulista, Departamento de Educação Física, Bauru, SP, Brasil.
\end{abstract}

\section{Correspondência:}

Rua Armando Pieroni, 3-39, Jardim Aeroporto, Bauru, SP, Brasil. 17015-050.

liagregomuniz@yahoo.com.br

\section{RESUMO}

Introdução: A aptidão física é imprescindível para policiais militares. Entretanto, apesar de a finalidade do treinamento militar ser o aperfeiçoamento de diferentes componentes da aptidão física, sua execução de forma não adequada pode associar-se ao desenvolvimento de lesões musculoesqueléticas. Objetivo: Verificar o efeito do treinamento físico militar sobre indicadores de aptidão física e incidência de lesões musculoesqueléticas em participantes do Curso de Formação de Soldados da Polícia Militar do Estado de São Paulo. Método: A amostra foi composta por 86 homens que foram submetidos ao Teste de Aptidão Física no início e depois de 54 semanas de treinamento. As informações sobre lesões foram obtidas com questionário específico. Resultados: Houve melhora significativa do volume máximo de oxigênio $\left(\mathrm{VO}_{2 \text { máx }}\right)$, resistência muscular localizada, força e velocidade ao final do treinamento. Entretanto, 45,3\% dos policiais sofreram uma ou mais lesões, sendo $65,6 \%$ delas concentradas nos membros inferiores, $18 \%$ nos membros superiores e 16,4\% no tronco e na cabeça. O nível osteoarticular concentrou 50,8\% das lesões, o nível músculo-ligamentar, 26,3\% e o tegumentar, 22,9\%. Conclusão: Apesar do resultado satisfatório em relação à aptidão física, a incidência de lesões entre os policiais foi elevada.

Descritores: aptidão física; militares; sistema musculoesquelético/lesões.

\section{ABSTRACT}

Introduction: Physical fitness is imperative for military police officers. However, although the purpose of military training is to improve different components of physical fitness, its inadequate performance may be associated with the development of musculoskeletal injuries. Objective: To verify the effect of military physical training on indicators of physical fitness and incidence of musculoskeletal injuries in participants of the Training Course of Military Police Officers in the State of São Paulo. Methods: The sample consisted of 86 men who were submitted to the Physical Fitness Test at the beginning and after 54 weeks of training. Information on injuries was obtained with a specific questionnaire. Results: There was significant improvement in maximum oxygen uptake $\left(\mathrm{VO}_{2 \text { max }}\right)$, localized muscle endurance, strength and velocity at the end of training. However, 45.3\% of the officers suffered one or more injuries, $65.6 \%$ of which were concentrated in the lower limbs, $18 \%$ in the upper limbs, and $16.4 \%$ in the torso and head. The osteoarticular level concentrated $50.8 \%$ of the injuries, the musculoligamentous level, 26.3\%, and the tegumentary level, 22.9\%. Conclusion: Despite the satisfactory results regarding physical fitness, the incidence of injuries among military police officers was high.

Keywords: physical fitness; military personnel; musculoskeletal system/injuries.

\section{RESUMEN}

Introducción: La buena aptitud física es indispensable para la policía militar. Si embargo, aunque el propósito del entrenamiento militar sea la mejora de los diferentes componentes de condición física, su ejecución inadecuada puede estar asociada con el desarrollo de lesiones musculoesqueléticas. Objetivo: Verificar el efecto del entrenamiento físico militar sobre los indicadores de aptitud física y la incidencia de lesiones musculoesqueléticas en los participantes del Curso de Entrenamiento de Soldados de la Policía Militar del Estado de São Paulo. Método: La muestra consistió en 86 hombres que fueron sometidos a la Prueba de Aptitud Física al inicio y después de 54 semanas de entrenamiento. La información sobre lesiones se obtuvo con cuestionario específico. Resultados: Hubo una mejora significativa del consumo máximo de oxígeno ( $\mathrm{VO}_{\text {máx }}$ ), la resistencia muscular localizada, fuerza y velocidad al final del entrenamiento. Sin embargo, el 45,3\% de los policías sufrieron una o más lesiones, siendo el 65,6\% concentrado en las extremidades inferiores, el $18 \%$ en las extremidades superiores y el 16,4\% en el torso y la cabeza. El nivel osteoarticular concentra el 50,8\% de las lesiones, el nivel musculoligamentoso, 26,3\% y el tegumentario, 22,9\%. Conclusión: A pesar de los resultados satisfactorios en términos de aptitud física, la incidencia de las lesiones entre los policías fue alto.

Descriptores: aptitud física; personal militar; sistema musculoesquelético/lesiones. 


\section{INTRODUÇÃO}

No Brasil, a polícia militar tem como missão o patrulhamento ostensivo e a preservação da ordem pública. Essa ocupação demanda exigências fisiológicas, recorrências de estados estressantes e rotinas extenuantes que podem provocar danos físicos e psicológicos a estes profissionais'. Teixeira e Pereira ${ }^{2}$ acrescentam que a estrutura e os objetivos do serviço militar exigem que seus participantes tenham boa condição física. Para monitorar o desempenho desses profissionais, são aplicadas baterias de testes físicos periodicamente, com o objetivo de verificar o nível físico dos militares, bem como incentivá-los à melhoria e à manutenção de suas capacidades'.

Assim níveis satisfatórios de aptidão física têm sido assinalados como fator de proteção para inúmeras condições mórbidas e como promotoras de melhor qualidade de vida. Da mesma forma, manter rotinas de atividades físicas de moderada a intensa se correlaciona negativamente com taxas de mortalidade por doenças cardiovasculares ${ }^{3,4}$.

A exigência dos militares em proporcionar segurança à comunidade tem por consequência, treinamentos e competições que frequentemente aumentam o risco de lesões do aparelho locomotor ${ }^{5}$. Minayo et al. ${ }^{6}$ relatam que a maioria dos pedidos de licença médica desses profissionais se deve a problemas ortopédicos por traumas físicos.

Após levantamento bibliográfico verificou-se que existem inúmeros estudos transversais que abordam a aptidão física de militares, mas em nosso meio, são escassas as pesquisas longitudinais sobre aptidão física e lesões musculoesqueléticas dessa população.

Face ao exposto, o objetivo da presente pesquisa foi verificar o efeito do treinamento físico militar sobre indicadores de aptidão física e incidência de lesões musculoesqueléticas em participantes do Curso de Formação de Soldados da Polícia Militar do Estado de São Paulo.

\section{MATERIAIS E MÉTODOS}

Trata-se de um estudo prospectivo quase-experimental com alunos do Curso de Formação de Soldados para integrar o efetivo da Polícia Militar (PM) de São Paulo.

A amostra foi composta por 86 homens em formação por 54 semanas junto ao Comando de Policiamento do Interior 4 (CPI-4) de Bauru, SP.

Para conhecimento e controle da condição física dos alunos, a PM aplica o Teste de Aptidão Física (TAF) no início e ao final do Curso. O desempenho obtido no final do TAF é transformado em nota classificatória, para fins de escolha das vagas oferecidas para a região pretendida.

Informações antropométricas como peso e estatura foram verificadas em ambos os momentos da avaliação, e o índice de massa corporal (IMC) utilizado para determinar a condição nutricional. Para tanto foi utilizada balança eletrônica da marca Bioland (modelo EB 9015) e estadiômetro portátil com precisão em milímetros (Sanny).

O TAF é constituído por quatro testes físicos, a saber: i) abdominal em um minuto (resistência muscular localizada): o avaliado em decúbito dorsal sobre um colchonete repete a maior quantidade possível de flexões do tronco sobre os membros inferiores ${ }^{7}$; ii) flexões dos cotovelos na barra fixa (força muscular): o indivíduo foi orientado a posicionar-se suspenso pelas mãos em posição pronada utilizando-se da barra fixa, sem mais nenhum apoio auxiliar para realizar a elevação do corpo por meio da flexão dos membros superiores até que o queixo ultrapassasse a barra fixa e, posteriormente, deveria retornar a posição inicial. Foram contabilizados apenas os movimentos realizados corretamente em 60 segundos ${ }^{8}$; iii) corrida de 50 metros (velocidade): a posição de saída foi com afastamento ântero posterior das pernas e com o pé da frente o mais próximo possível da faixa. Ao comando de voz o cronômetro foi acionado e parado quando o avaliado ultrapassou o peito na faixa de chegada. Foi registrado o tempo de percurso dos 50 metros com precisão de centésimos de segundo9; iv) teste de 12 minutos (condição cardiorrespiratória): os avaliados percorreram a maior distância possível, nesse intervalo de tempo ${ }^{10}$.

Os testes foram aplicados pelo coordenador da pesquisa, que acumula a habilitação em Educação Física e a patente de tenente coronel da instituição.

Para o diagnóstico da condição nutricional foram empregados os pontos de corte propostos pela Organização Mundial da Saúde ${ }^{11}$.

Para levantamento das informações sobre aspectos comportamentais e relacionados às rotinas de atividade física um inquérito validado foi respondido individualmente por cada aluno do Curso.

Para verificar o nível de atividade física foi indagado aos participantes se eles praticavam atividades físicas no tempo livre. Foram classificados de acordo com as frequências de prática semanal. Quando a resposta foi não praticar exercícios ou apenas uma vez na semana foram considerados fisicamente inativos no tempo livre; se praticavam atividade física duas vezes na semana foram identificados como insuficientemente ativos; e, se realizavam exercícios físicos três vezes na semana ou mais foram considerados ativos. Quanto ao consumo de álcool e tabaco foi registrado apenas se faziam uso regular ou não.

Para identificar a ocorrência de lesões musculoesqueléticas ao longo do curso foi utilizado um formulário, onde foi informado, o tipo de lesão, a região corporal e a causa situacional (momento do treinamento que a lesão ocorreu). Os participantes da pesquisa relataram somente as lesões que ocorreram no momento do treinamento. Ambos os instrumentos utilizados para a coleta dos dados mencionados acima foram validados em investigações anteriores ${ }^{12,13}$.

\section{Procedimentos de campo}

Foi aplicado o TAF no início e no final do Curso de Formação. No intervalo do pré e pós-teste foi realizado o treinamento físico.

As aulas foram ofertadas duas vezes por semana com sessões de 90 minutos cada, sempre em dias alternados. Cada sessão compreendia as seguintes etapas: i) Trabalho aeróbio em intensidade leve e aumento gradativo até alcançar o grau moderado, geralmente realizado sob a forma de corrida (45 minutos); ii) Volta a calma com caminhada leve (15 minutos); iii) Exercícios de flexibilidade e alongamento (30 minutos). Sob a regência de outro professor, acontecia também uma terceira sessão semanal de 60 minutos dedicada ao aprendizado de técnicas de defesa pessoal, onde os exercícios localizados eram trabalhados sistematicamente. Ao final de 54 semanas foram totalizadas 220 horas de condicionamento físico, sendo 170 sob a regência do coordenador do estudo e 50 horas pelo professor de defesa pessoal.

O programa de atividades físicas realizadas durante a formação de soldados é parte de um processo periódico da corporação que enfoca conteúdos teóricos e práticos voltados ao exercício profissional. Sobre os conteúdos práticos, especificamente aqueles que se destinam a melhorar o condicionamento físico e a defesa pessoal, o Comando operacional do batalhão indicou a necessidade de avaliar os efeitos do treinamento físico sobre os indicadores de performance desses alunos, solicitando a realização da presente investigação.

A Faculdade de Ciências da Unesp, dispensa a aprovação do Comitê de Ética para o presente estudo. Todos os procedimentos de pesquisa empregados atenderam as exigências da Resolução específica do Conselho Nacional de Saúde (no 196/96) que fixam Diretrizes e Normas para pesquisa envolvendo seres humanos. Todos participantes consentiram sua participação na pesquisa.

\section{Procedimentos estatísticos}

Para apresentação dos dados foram utilizadas distribuições de frequências absoluta e relativa (\%) e para verificar associações o Teste t pareado (com nível de significância: $p<0,05$ ). 


\section{RESULTADOS}

Foram avaliados 86 indivíduos do sexo masculino, com média de idade de $24 \pm 3$ anos, de classe social média-baixa e, a maioria, com o ensino médio concluído.

Observou-se que 15,1 e 39,5\% dos investigados consomem álcool e fumam regularmente, respectivamente. No tempo livre, 54,7\% realizam exercícios físicos com frequência mínima de três vezes por semana e 26,7\% estavam inativos nos últimos seis meses. Durante os 12 meses da formação de soldados 45,3\% sofreram algum tipo de lesão musculoesquelética.

Foi observada melhora significativa entre as avaliações inicial e final para a resistência aeróbica, resistência muscular localizada, força muscular, velocidade e escore final dos testes (Tabela 1).

Quando avaliados por segmento corporal, 65,6\% de todas as lesões se concentraram nos membros inferiores, $18 \%$ nos superiores e $16,4 \%$ no tronco/cabeça (Figura 1).

Distribuição de frequência das lesões por segmentos corporais encontra-se descrita na Figura 2. No segmento tronco/cabeça (16,4\%), as regiões corporais afetadas foram 13,1\% na cabeça (corte 9,8\% e abrasão 3,3\%), 1,6\% ombro (luxação) e 1,6\% costas (distensão).

A frequência de lesões no braço foi de 8,2\% (abrasão 3,2\% e fratura 5\%), 1,6\% no cotovelo (entorse), 5\% no antebraço (fratura) e 3,2\% nas mãos (abrasão 1,6\% e entorse 1,6\%). A fratura foi um agravo com alta frequência, com 10\% apenas nas regiões do braço e antebraço.

O tornozelo $(24,6 \%)$ e o joelho $(21,3 \%)$ foram as regiões mais afetadas. No tornozelo predominaram os entorses $9,8 \%$ e luxações $8,2 \%$; o joelho concentrou 10 diferentes tipos de lesões sem predominância de nenhuma. Em relação às outras regiões corporais a coxa corresponde a $5 \%$, perna $6,5 \%$ e os pés $8,2 \%$.

O plano osteoarticular foi o que concentrou a maior taxa de lesões

Tabela 1. Média, desvio-padrão e resultados do Teste $t$ pareado das variáveis antropométricas, da condição física (TAF) dos alunos e total da avaliação do TAF, segundo respectivos momentos de avaliação.

\begin{tabular}{c|c|c|c}
\hline Variável & Pré & Pós & $\begin{array}{c}\text { Teste } \boldsymbol{t} \\
\text { (p-valor) }\end{array}$ \\
\hline Peso $(\mathrm{kg})$ & $74,2 \pm 9,9$ & $72,3+8,5$ & $1,350(0,179)$ \\
\hline IMC $\left(\mathrm{kg} / \mathrm{m}^{2}\right)$ & $24 \pm 2,6$ & $23,4+2,2$ & $1,634(0,104)$ \\
\hline VO máx. $\left(\mathrm{ml} / \mathrm{kg} / \mathrm{min}^{-1}\right)$ & $44,9 \pm 5,3$ & $53,1+3,6$ & $-11,869(0,001)$ \\
\hline RML (repetições) & $39,6 \pm 3,7$ & $47,5+3,9$ & $-13,628(0,001)$ \\
\hline Força muscular (repetições) & $6,3 \pm 3,5$ & $8,6+2,9$ & $-4,693(0,001)$ \\
\hline $\begin{array}{c}\text { Velocidade em 50 } \\
\text { metros (segundos) }\end{array}$ & $7,2 \pm 0,4$ & $6,8+0,3$ & $7,419(0,001)$ \\
\hline Escore final & $278,3 \pm 50,7$ & $368,7+26$ & $-14,713(0,001)$ \\
\hline
\end{tabular}

IMC: Indice de Massa Corporal; VO2 máx: Volume Máximo de Oxigênio; RML: Resistência Muscular Localizada.

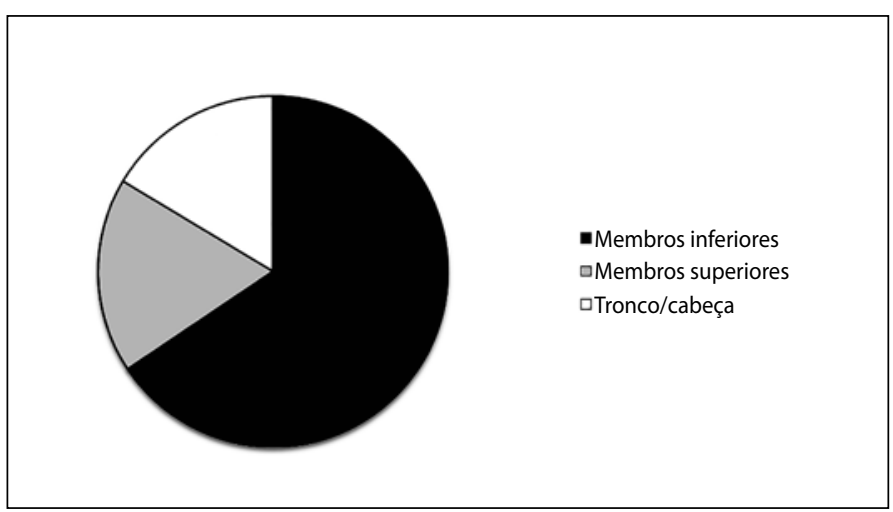

Figura 1. Distribuição de frequência dos agravos referidos pelos alunos do Curso de Formação de Soldados do Comando de Policiamento do Interior 4 - Bauru por segmento corporal.
(50,8\%), no qual o entorse foi o referido, com 21,3\% de todos os episódios. No plano músculo ligamentar ocorreram $26,3 \%$ das lesões e, entre estas, distensão $(11,5 \%)$ e tendinite $(8,2 \%)$ foram as mais frequentes. No plano tegumentar (22,9\%), o corte $(57,1 \%)$ foi o mais referido (Tabela 2 ).

Entre os 86 participantes da pesquisa, nas 54 semanas de trabalho foram observadas 61 lesões que provocaram incapacidade temporária para a realização de exercícios físicos e/ou funções ocupacionais, implicando, em alguns casos, na limitação para a execução de atividades da vida diária.

\section{DISCUSSÃO}

No presente trabalho verificou-se melhora significativa de todas as variáveis relacionadas à aptidão física após 54 semanas de intervenção, contudo quase metade dos participantes sofreu alguma lesão, sendo as mais frequentes nos membros inferiores, com predominância dos tornozelos e joelhos.

Verificou-se que 39,5\% dos policiais referiram ser tabagistas. Ferreira et al. ${ }^{14}$, ao investigarem estilo de vida de policiais militares, encontraram que $12 \%$ eram fumantes, frequência inferior à observada na atual pesquisa. Esse resultado é preocupante, porque o tabagismo é uma doença classificada pela CID $^{15}$ no grupo de transtornos mentais e comportamentos associados ao uso de substâncias psicoativas. Munnoch e Bridger ${ }^{16}$ observaram que a incidência de lesões entre fumantes é duas vezes maior do que entre não fumantes.

O consumo regular de álcool foi informado por 15,1\% dos avaliados. Estudo anterior indicou que militares que fazem uso regular de álcool são mais propensos ao acometimento por doenças, hospitalizações, acidentes, mortes por afogamento, automutilação e suicídio ${ }^{17}$. Para Souza et al. ${ }^{18}$ esse assunto é controverso e inconclusivo, porque algumas teorias defendem que o consumo de substâncias psicoativas podem acalmar e aliviar as tensões, ameaças e afetos negativos provocados pelas duras condições de trabalho e seus fatores geradores de ansiedade, enquanto

Tabela 2. Distribuição de frequência absoluta e relativa dos agravos referidos pelos alunos do Curso de Formação de Soldados do Comando de Policiamento do Interior 4 - Bauru, segundo plano e natureza da lesão.

\begin{tabular}{c|c|c|c}
\hline Plano da lesão & $\begin{array}{c}\text { Natureza } \\
\text { da lesão }\end{array}$ & $\mathbf{N}(\%)$ & \% do total de lesões \\
\hline Tegumentar & Corte & $8(57,1)$ & 13,1 \\
\hline Músculo-ligamentar & Abrasão & $6(42,9)$ & 9,8 \\
\hline & Distensão & $7(43,7)$ & 11,5 \\
\hline & Contusão & $4(25)$ & 6,6 \\
\hline Ósteo-articular & Tendinite & $5(31,3)$ & 8,2 \\
\hline & Entorse & $13(42)$ & 21,3 \\
\hline & Luxação & $8(25,8)$ & 13,1 \\
\hline & Fratura & $10(32,2)$ & 16,4 \\
\hline
\end{tabular}

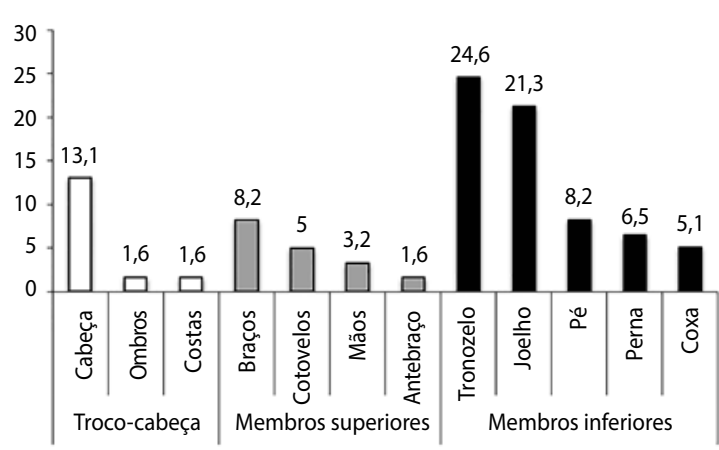

Figura 2. Distribuição de frequência dos agravos referidos pelos alunos do Curso de Formação de Soldados do Comando de Policiamento do Interior 4 - Bauru por partes em cada segmento corporal. 
outras consideram que o consumo crônico induz à ansiedade, ataque e transtorno de pânico, "agorafobia", entre outros transtornos mentais.

Em relação à prática de atividades físicas, foi observado que mais de $45 \%$ dos investigados relataram não praticar qualquer atividade física no tempo livre. Entre os motivos que dificultam policiais se engajarem em atividades físicas no tempo livre encontram-se: compromissos familiares, jornada de trabalho, falta de equipamento, ambiente inseguro, falta de companhia, tarefas domésticas e falta de recursos financeiros ${ }^{19}$.

Quanto às variáveis de aptidão física, os investigados apresentaram evolução para $\mathrm{VO}_{2}$ máximo (44,9 \pm 5,3 vs. 53,1 $\left.\pm 3,6 \mathrm{ml} / \mathrm{kg} / \mathrm{min}-1 ; p<0,05\right)$, valores inferiores aos encontrados em pesquisa com 50.523 militares do exército brasileiro (54 a $57 \mathrm{ml} / \mathrm{kg} / \mathrm{min}-1)^{20}$.

Com relação à força muscular, nossos resultados corroboram com Vargas et al. ${ }^{21}$, que verificaram melhora significativa no teste de força (6,3 \pm 3,5 repetições vs. 8,6 $\pm 2,9 ; p=0,001$ ). Para resistência muscular localizada, os participantes encontravam-se classificados como "acima da média" no início do treinamento e passaram para o padrão "excelente" após o treinamento. A melhora dessas duas capacidades físicas é imprescindível, porque policiais necessitam realizar tarefas diversas suportando o peso do armamento. Além disso, musculatura fortalecida pode reduzir a probabilidade de ocorrência de entorses, rupturas musculares e outras lesões características de quem pratica atividade física ocupacional de intensidade elevada².

Em se tratando da velocidade, Berria et al. ${ }^{22}$ aplicaram o teste de corrida de 50 metros em policiais da região sul do país e obtiveram média de 7,9 $\pm 1,3$ segundos. Na presente investigação encontramos médias semelhantes (7,2 \pm 0,4 segundos no pré-teste e 6,8 $\pm 0,3$ segundos no pós-teste). Considerando os testes de aptidão física de maneira geral, os participantes da presente pesquisa obtiveram melhora significativa entre o inicio e o final da avaliação. Sobre esse assunto, Matos et al..$^{23}$ comentam que boa aptidão física é importante para o estado de prontidão dos militares e proporciona melhores condições para suportar a rotina diária. Entretanto, apesar da melhora de todos os componentes da aptidão física, 45,3\% ( $n=39$ ) apresentaram 61 lesões, o que resultou em 1,5 agravos por pessoa no período de 54 semanas de formação. Esse percentual se aproxima das taxas encontradas em outros estudos, que variam de 34\% a 46\%24,25. Segundo pesquisa americana, variáveis que contribuem para maior risco de lesão entre militares são o tabagismo e baixo engajamento com a prática regular de exercícios físicos ou esportes antes do período de treinamento ${ }^{20}$.

Esses resultados podem se agravar mais após esses recrutas se efetivarem, pois, segundo Moore et al. ${ }^{26}$, historicamente, lesões musculoesqueléticas são a principal causa de entrada em ambulatório para o tratamento de militares. No Reino Unido, em 2010, foram registradas aproximadamente 2,5 milhões de visitas ao ambulatório por lesões musculoesqueléticas, onde os membros inferiores se destacaram com as maiores frequências, representando um gasto de 548 mil dólares na assistência direta ao paciente.

Entre os avaliados, 65,6\% das lesões ocorreram nos membros inferiores, sendo o entorse de tornozelo a lesão mais frequente. Outros estudos investigando militares também encontraram os membros inferiores como a região com as maiores frequências de agravos ${ }^{9,27}$. Nesta direção, Guisande et al. ${ }^{28}$ analisaram as forças de reação do solo na marcha militar de soldados do exército brasileiro e concluíram que há uma força vertical transiente que não está normalmente presente no padrão de marcha normal. Provavelmente esta força resulta do padrão de marcha militar, com o pé atingindo o solo de forma breve e forte. Baseado nisso, estudo prospectivo realizado com soldados americanos em treinamento encontrou que tênis de corrida confeccionado de acordo com a forma dos pés dos soldados contribuíram para reduzir a ocorrência de lesões ${ }^{29}$. No presente estudo, todo o treinamento aeróbico foi realizado com corrida em pavimento asfáltico. Estudos com corredores de rua apontam que lesões decorrentes desse tipo de treinamento, também apontam o joelho como a região mais afetada ${ }^{30}$.

Destaca-se como limitação do estudo a obtenção de dados por auto relato, o que não permitiu aprofundar questões específicas relacionadas às lesões. Outra restrição foi a ausência de grupo controle que poderia esclarecer se as melhoras nas variáveis analisadas foram exclusivamente devidas ao treinamento ou se a prática de atividades físicas realizadas fora do centro de treinamento teria alguma influência.

Apesar dos resultados terem sido satisfatórios em relação à aptidão física, a incidência de lesões entre os recrutas foi elevada, podendo prejudicar o desempenho na profissão. O treinamento resistido adequado e a utilização de exercícios de flexibilidade e relaxamento, especialmente antes de trabalho intenso, podem contribuir para reduzir o número de lesões. Outros cuidados que podem prevenir os traumatismos, como variações nas técnicas, trocas da velocidade de movimentos, corridas sobre superfícies macias (grama, areia), planejamento cuidadoso do trabalho aeróbio e utilização de calçado especial que assegure estabilidade e suavize a força de apoio são sugeridos.

\section{AGRADECIMENTOS}

Os autores agradecem a Polícia Militar do Estado de São Paulo, a CNPq (Conselho Nacional de Desenvolvimento Tecnológico e Científico) e a CAPES (Coordenação de Aperfeiçoamento de Pessoal de Nível Superior) pelo apoio dado a esta pesquisa.

Todos os autores declararam não haver qualquer potencial conflito de interesses referente a este artigo.

CONTRIBUIÇÕES DE AUTORES: Cada autor contribuiu individual e significativamente para o desenvolvimento do manuscrito. MSO (0000-0002-4430-8889)* e HLM (00000001-6639-1532)* foram os autores que contribuíram na concepção e desenho da pesquisa. LGMA (0000-0002-2885-7730)*e MS realizaram a pesquisa bibliográfica. LGMA BCT (0000-0002-1314-6258)* e HLM foram os principais colaboradores na redação do manuscrito. MS realizou a coleta de dados. BCT, HLM realizaram a análise dos dados. Todos os autores revisaram e aprovaram a versão final do trabalho. ${ }^{*}$ ORCID (Open Researcher and Contributor ID).

\section{REFERÊNCIAS}

1. Silva R, Schlichting AM, Schlichting JP, Gutierres Filho PJ, Adami F, Silva A. Aspectos relacionados à qualidade de vida e atividade física de policiais militares de Santa Catarina-Brasil. Motricidade. 2012;8(3):81-9.

2. Teixeira CS, Pereira EF. Physical fitness, age and nutritional status of military personnel. Arq Bras Cardiol. 2010;94(4):438-43

3. McGuire S. Centers for Disease Control and Prevention. State indicator report on Physical Activity, 2014. Atlanta, GA: U.S. Department of Health and Human Services; 2014. Adv Nutr. 2014;5(6):762-3.

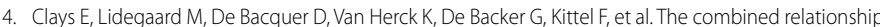
of occupational and leisure-time physical activity with all-cause mortality among men, accounting for physical fitness. Am J Epidemiol. 2014;179(5):559-66.

5. Calansan DA, Borin G, Peixoto GT. Lesões musculoesqueléticas em policiais militares. Rev Bras Med Esporte. 2013;19(6):415-8
6. Minayo MCS, Assis SG, Oliveira RVC. Impacto das atividades profissionais na saúde física e mental dos policiais civis e militares do Rio de Janeiro. Ciênc Saúde Coletiva. 2011;16(4):2199-209.

7. Johnson BL, Nelson JK. Pratical measurements for evaluation in physical education. 4th ed. Edina Burgerss; 1986

8. Pollock ML, Wilmore JH. Exercícios na saúde e na doença: avaliação e prescrição para prevenção e reabilitação. 2a. ed. Rio de Janeiro: Medis; 1993.

9. AAHPERD. Health related physical fitness test manual. Reston, American Alliance for Health, Physical Education, Recreation and Dance; 1980.

10. Cooper KH. A means of assessing maximal oxygen intake. Correlation between field and treadmil testing. JAMA. 1968;203(3):201-4.

11. Obesity: preventing and managing the global epidemic. Report of a WHO consultation. World Health 
Organ Tech Rep Ser. 2000;894:i-xii, 1-253.

12. Monteiro HL, Romero LR, Padovani CR. A geração do futuro: classe social, níveis de atividade física, desempenho motor, hábitos e morbidade de escolares de Segundo Grau do Município de Bauru - SP. Rev Bras Cienc Esporte. 1999;21(1):826-31.

13. Grego LG, Monteiro HL, Padovani CR, Gonçalves A. As lesões na dança: estudo transversal híbrido em academias da cidade de Bauru - SP. Rev Bras Med Esporte 1999;5(2):41-54

14. Ferreira DKS, Bonfim C, Augusto LGS. Fatores associados ao estilo de vida de policiais militares. Ciênc Saúde Coletiva. 2011;16(8):3403-12

15. . Organização Mundial da Saúde. CID-10: Classificação estatística internacional de doenças e problemas relacionados a saúde. 10a. ed. São Paulo: EDUSP; 2009.

16. Munnoch K, Bridger RS. Smoking and injury in Royal Marines'training. Occup Med (Lond). 2007;57(3):214-6.

17. Fear NT, Iversen A, Meltzer H, Workman L, Hull L, Greenberg N, et al. Patterns of drinking in the UK Armed Forces. Addiction. 2007;102(11):1749-59.

18. Souza ER, Schenker M, Constantino B, Correia BSC. Consumo de substâncias lícitas e ilícitas por policiais da cidade do Rio de Janeiro. Ciênc Saúde Coletiva. 2013;18(3):667-76.

19. Jesus GM, Jesus EFA. Nível de atividade física e barreiras percebidas para a prática de atividades físicas entre policiais militares. Rev Bras Ciênc Esporte. 2012;34(2):433-48.

20. Oliveira EAM, Anjos LA. Medidas antropométricas segundo aptidão cardiorrespiratória em militares da ativa, Brasil. Rev Saúde Pública. 2008;42(2):217-23.

21. Vargas LM, Moleta T, Pilatti LA. Diferença da aptidão física relacionada ao desempenho entre soldados de elite e convencionais do exército brasileiro. Conexões. 2013;11(2):148-67.

22. Berria J, Daronco LSE, Bevilacqua LA. Aptidão motora e capacidade para o trabalho de policiais militares do batalhão de operações especiais. Salusvita. 2011;31(2):89-104.

23. Matos DG, Salgueiro RS, Mazini Filho ML, Rodrigues BM, Aidar FJ. Lima RJP. Perfil evolutivo do condicionamento aeróbio e da força em policiais militares Rev Bras Cienc Saúde. 2010; 8(25):14-20.

24. Knapik JJ, Graham B, Cobbs J, Thompson D, Steelman R, Jones BH. A prospective investigation of injury incidence and injury risk factors among Army recruits in military police training. BMC Musculoskele Disord. 2013;14:32.

25. Jones BH, Cowan DN, Tomlinson JP, Robinson JR, Polly DW, Frykman PN. Epidemiology of injuries associated with physical training among young men in the army. Med Sci Sports Exerc. 1993;25(2):197-203.

26. Moore JH, Goffar SL, Teyhen DS, Pendergrass TL, Childs JD, Ficke JR. The role of U.S. military physical therapists during recent combat campaigns. Phys Ther. 2013;93(9):1268-75.

27. Hauret KG, Jones BH, Bullock SH, Canham-Chervak M, Canada S. Musculoskeletal injuries description of an under-recognized injury problem among military personnel. Am J Prev Med. 2010;38(Suppl 1):S61-70

28. Guisande TP, Amadio JC, Amadio AC, Mochizuki L. As forças transientes durante a marcha militar. Rev Bras Med Esporte. 2013;19(6):390-3.

29. Knapik JJ, Trone DW, Swedler DI, Villasenor A, Bullock SH, Schmied E, et al. Injury reduction effectiveness of assigning running shoes based on plantar shape in Marine Corps basic training. Am J Sports Med. 2010;38(9):1759-67.

30. Van Middelkoop M, Kolkman J, Van Ochten J, Bierma-Zeinstra SM, Koes B. Prevalence and incidence of lower extremity injuries in male marathon runners. Scand J Med Sci Sports. 2008;18(2):140-4. 